\title{
THE GROUPS IN WHICH EVERY SUBGROUP IS EITHER ABELIAN
}

\section{OR HAMILTONIAN*}

BY

\section{G. A. MILLER}

The non-abelian groups which contain only abelian subgroups have been determined and many of their fundamental properties have been noted. $\dagger$ The present paper is devoted to a determination and a study of the non-abelian groups which involve only abelian and hamiltonian subgroups. It will be found that there are only two exceptions to the following theorem : If a group contains at least one hamiltonian subgroup and if all its subgroups are either abelian or hamiltonian the group itself is hamiltonian. The two exceptional groups are of orders 16 and 24 respectively, and are completely determined in the following paragraphs.

Let $G$ represent any group in which every subgroup is either abelian or hamiltonian and suppose that it is represented as a transitive substitution group of the lowest possible degree. If it is imprimitive its systems of imprimitivity are permuted according to some primitive group. To prove that $G$ is solvable it is only necessary to prove that this primitive group is composite since it may be simply isomorphic with $G$ and since every subgroup of $G$ is solvable. The primitive group in question is of class $n-1, n$ being its degree, since two of its maximal subgroups of degree $n-1$ can have only the identity in common. $\ddagger$ As every group of class $n-1$ and degree $n$ is composite $G$ is solvable.

In the preceding paragraph it is not assumed that $G$ necessarily contains a hamiltonian subgroup. This assumption may, however, be made since the nonabelian groups in which every subgroup is abelian are known. Hence we shall assume in what follows that $G$ contains at least one hamiltonian subgroup. Its order can therefore not be the power of an odd prime number. We shall consider all the possible $G$ 's under two headings as their orders are a power of 2 or involve more than one prime factor.

\footnotetext{
* Presented to the Society October 27, 1906. Received for publication October 19, 1906.

† Miller and Moreno, Transactions of the Amerioan Mathematical Society, vol. 4 (1903), p. 398.

$\ddagger$ Loc. cit.
} 


\section{$\S 1$. The order of $G$ is $2^{m}$.}

Since $G$ involves at least one hamiltonian subgroup it must involve a hamiltonian subgroup $(H)$ which is composed of half the operators of $G$. Since $H$ is hamiltonian, three-fourths of its operators are non-invariant, of order four, and have a common square.* The remaining one-fourth are the invariant operators of $H$ and they constitute an abelian subgroup $(K)$ of type $(1,1,1, \cdots)$. With respect to $K, H$ is isomorphic to the four-group. There is at least one subgroup ( $\left.H^{\prime}\right)$ in $H$ which involves $K$ and one-third of the operators of order four contained in $H$ and is invariant under $G$. The subgroup $H^{\prime}$ is abelian and of type $(2,1,1, \cdots)$.

As $H$ does not contain any operator whose order exceeds 4 there can be no operator whose order exceeds 8 in $G$. Moreover, every operator of order 2 is invariant under $G$. This results from the following considerations. Any operator of $G$ together with $K$ generate a subgroup which is either abelian or hamiltonian since this operator and $K$ do not generate $G$. As every operator of order 2 in a hamiltonian group is invariant this proves that every operator of $K$ is invariant under $G$. If any other operator of order 2 were not commutative with some operator of order 4 in $H$ the latter operator could not be in $H^{\prime}$ since a hamiltonian group involves no non-invariant operator of order 2 . That is, each operator of $H^{\prime}$ is commutative with every operator of order 2 contained in $G$. If an operator of order 2 were not commutative with any other operator of order 4 in $H$ it would transform this operator into itself multiplied by an operator of order 2 contained in $H$. Hence the order of $G$ could not exceed 16. As the groups of order 16 are so well known it seems unnecessary to go into further details to establish the theorem in question.

We shall now prove that there is one and only one $G$ which involves operators of order 8. As an operator $(t)$ of order 8 in $G$ is commutative with all the operators of $K$ and has its square in $H^{\prime}$ it must be commutative with every operator of $H^{\prime}$. It cannot be commutative with every operator of $H$ since $t^{2}$ transforms some of these operators into their inverses. As $t$ transforms an operator of order 4 in $H$ into itself multiplied by an operator of order 4 which is commutative with $t \dagger$, the group generated by $t$ and any operator $(s)$ of order 4 in $H$ which is not commutative with $t$ is of order 16 or 32 . This group must coincide with $G$ since it is neither abelian nor hamiltonian.

We proceed to prove that the order of the group $\{s, t\}$ generated by $s$ and $t$ cannot be 32 . If this order were $32, t^{2}$ would not be in the group of order 8 generated by $s$ and the commutative subgroup of $\{s, t\}$. This group and $t^{2}$ would therefore generate the hamiltonian group of order 16 and $G$ would involve the abelian group of type $(3,1)$. This abelian subgroup would involve all the

\footnotetext{
* Bulletin of the American Mathematical Society, vol. 4 (1898), p. 510.

$\dagger$ Bulletin of the American Mathematical Society, vol. 7 (1901), p. 350.
} 
operators of order 8 contained in $G$ and two of the remaining operators of order 4 would generate a non-abelian group of order 16 involving a commutator subgroup of order 4. As such a subgroup could not occur in $G$ it has been proved that the order of $G$ cannot exceed 16. It may be remarked that this result could be directly obtained from the properties of the known groups of order 32.* As the groups of order 16 are known we may state the result as follows: There is one and only one group of order $2^{m}$ which involves operators whose orders exceed four and satisfies the additional conditions that every subgroup is either abelian or hamiltonian and that at least one subgroup is hamiltonian. This is the group of order 16 which contains a cyclic subgroup of order 8 while the remaining operators are of order 4 and transform the operators of this cyclic subgroup into their inverses.

Half of the operators of $G$ which are not also in $H$ transform the operators of order 4 in $H^{\prime}$ into their inverses while the other half are commutative with these operators. Hence $H^{\prime}$ is contained in two hamiltonian and one abelian subgroup whose order is half the order of $G$. We proceed to prove that $G$ contains operators of order 2 which are not in $H$ whenever its order exceeds 16. Let $t_{1}$ represent an operator of $G$ which is not in $H$ and which transforms into their inverses the operators of order 4 in $H^{\prime}$. The operators of order 4 in $H$ and $t_{1}$ have a common square. The group generated by $t_{1}$ and an operator of $H$ which is not contained in $H$ is at most of order 16 . It could not be of this order since two operators of a hamiltonian group cannot generate a group of order 16. From this it follows that $t_{1}$ is commutative with half the operators of $H$ and hence with operators of order 4 in $H$. As the product of $t_{1}$ into such an operator is of order $2, G$ is the direct product of $H$ and an operator of order 2. That is, If every subgroup of a group of order $2^{m}, m>4$, is either abelian or hamiltonian and if there is at least one hamiltonian subgroup, the entire group is hamiltonian.

It remains to consider the groups of order 16 . If such a group contains a hamiltonian subgroup it must be the quaternion group. As every group of order 16 contains an abelian subgroup of order 8 and as the groups under consideration do not involve any operators of order 8 , it follows that $G$ would have to contain operators of order 2 in addition to the one contained in the quaternion subgroup. As such an operator has to be commutative with each operator in the quaternion subgroup $G$ itself is the hamiltonian group of order 16, and it results that the group of order 16 which was considered above is the only nonhamiltonian group of order $2^{m}$ in which every subgroup is either abelian or hamiltonian and in which at least one subgroup is hamiltonian. With this single exception the hamiltonian groups are the only ones involving hamiltonian subgroups, but no other non-abelian subgroups.

\footnotetext{
*Quarterly Journal of Mathematics, vol. 28 (1896), p. 233.
} 


\section{§ 2. The order of $G$ is divisible by at least two different prime numbers.}

Since $G$ involves at least one hamiltonian subgroup its order is divisible by 8. It contains an invariant subgroup $H$ of prime index $(p)$ as it is solvable. This subgroup is the direct product of its own Sylow subgroups since it is either hamiltonian or abelian, and the order of every operator of $G$ which is not also in $H$ is divisible by $p$. Let $t$ represent one of these operators, so that $t^{p}$ is in $H$ but $t$ is not in $H$. Each of the Sylow subgroups of $H$ is transformed into itself by $t$.

When $p=2, H$ involves at least two different Sylow subgroups and $t$ is commutative with all the operators of odd order contained in $H$. The group generated by $t$ and the Sylow subgroup of order $2^{m}$ contained in $H$ is hamiltonian since it is a Sylow subgroup of $G$. As all of its operators are commutative with every operator of odd order in $H, G$ is the direct product of its Sylow subgroups whenever it contains an invariant subgroup of half its order. The Sylow subgroup of order $2^{m+1}$ is hamiltonian while all the others are abelian. Moreover, any such direct product is a group of the required kind. It remains to consider the cases when $p$ is odd.

When $p>2$ the Sylow subgroup of order $2^{m}$ contained in $H$ is hamiltonian. When $H$ involves more than one Sylow subgroup, $t$ is commutative with every operator of $H$ since it is commutative with all the operators in its Sylow subgroups. Hence $G$ is the direct product of its Sylow subgroups whenever the order of $H$ is divisible by at least two different prime numbers. It remains only to consider the case when $H$ is the hamiltonian group of $2^{m}$ and $p>2$.

Since $H$ contains only three abelian subgroups of order $2^{m-1}, t$ would have to transform each of these into itself if $p>3$. As the group generated by $t$ and such a subgroup would be abelian, $t$ would again be commutative with every operator of $H$ and we have proved that if the order of $G$ is not of the form $2^{m} .3$ $G$ is always the direct product of an abelian group of odd order and a hamiltonian group of order $2^{m}$. We shall therefore assume that the order of $G$ is $2^{m} .3$ and consider the possible value of $m$.

The order of the subgroup generated by three operators of order 4 in $H$ cannot exceed 16. Hence if we assume that $t$ is not commutative with every operator of order 4 in $H$ it transforms a subgroup of $H$ whose order cannot exceed 16 into itself. Since the group generated by $t$ and this subgroup is neither abelian nor hamiltonian the order of $G$ cannot exceed 48 unless it is the direct product of its Sylow subgroups. We may assume that $t$ does not transform each of the three abelian subgroups of order $2^{m-1}$ into itself, otherwise it would be commutative with each operator of $H$, and hence the group which the three conjugate operators of order 4 generate is the hamiltonian group of order 16 or the quaternion group. As the former contains exactly four quaternion 
subgroups $t$ and this quaternion subgroup generate the group of order 24 which has no subgroup of order 12 .

The main results of this section may be stated as follows: If a group contains at least one hamiltonian subgroup and if all its subgroups are either abelian or hamiltonian it is the direct product of the hamiltonian group of order $2^{m}$ and an abelian group of odd order, unless it is the group of order 24 which does not contain a subgroup of order 12. Hence there are only two non-hamiltonian groups which contain at least one hamiltonian subgroup and whose other subgroups are either abelian or hamiltonian. The orders of these wellknown groups are 16 and 24 respectively. While there is an infinite number of non-abelian groups in which every subgroup is abelian, there are only two other non-hamiltonian groups in which every non-abelian subgroup is hamiltonian. 